\title{
Intervenções benéficas no pré-natal para prevenção da mortalidade materna
}

Beneficial interventions for maternal mortality prevention in the prenatal period

Iracema de Mattos Paranhos Calderon ${ }^{1}$, José Guilherme Cecatti², Carlos Eduardo Pereira Vega ${ }^{3}$

\section{RESUM0}

A razão de mortalidade materna (MM) é indicador da qualidade de saúde, influenciada diretamente pelo grau de desenvolvimento econômico-cultural-tecnológico de um país. Os dados oficiais de MM no Brasil, ainda que subestimados, sinalizam a falta de qualidade dos serviços de assistência à gestação, parto e puerpério. Esta característica é comum entre os países em desenvolvimento, onde estão as gestantes mais necessitadas e com maior dificuldade de acesso a assistência de qualidade. A assistência pré-natal não pode prevenir as principais complicações do parto, causas importantes de MM, mas algumas intervenções no pré-natal poderão favorecer o prognóstico materno e prevenir a MM. Neste contexto, o artigo faz uma atualização, embasada em evidências científicas, sobre intervenções efetivas no pré-natal para prevenção da mortalidade materna. As estratégias mais importantes constituem um tripé, com intervenções específicas relacionadas a promoção da saúde materna, prevenção dos riscos e garantia de suporte nutricional durante a gestação, além de critérios para investigação do risco gestacional e inclusão da gestante no componente básico do modelo de assistência pré-natal. Finaliza com a definição de prioridades na prevenção de MM relacionada à eclâmpsia/pré-eclâmpsia e reforça a importância da normatização dos sistemas de referência para os casos de emergência obstétrica.

PALAVRAS-CHAVE: Mortalidade materna/prevenção e controle; Assistência pré-natal; Saúde da mulher

\section{ABSTRACT}

Maternal mortality rate (MM) is a health quality indicator that is directly influenced by the economic, cultural and technological level of a country. Official data of MM in Brazil, although underestimated, point to the lack of quality in pregnancy, childbirth and puerperium care services. This characteristic is common in developing countries, where poorer pregnant women as well as those facing greater difficulty to quality care access are found. Prenatal care cannot prevent major childbirth complications, which are important causes of MM; however, some interventions during the prenatal period can favor maternal prognosis and prevent MM. In this setting, this study brings a scientifically based update concerning effective interventions for maternal mortality prevention during the prenatal period. The most important strategies consist of a tripod with specific interventions related to maternal health promotion, risk prevention and assurance of nutritional support during gestation, in addition to criteria to investigate gestational risk and inclusion of the pregnant woman in the basic component of the prenatal care model. It ends with the definition of priorities in the prevention of MM related to eclampsia/preeclampsia and reinforces the importance of normalization of reference systems for obstetric emergency cases.

KEYWORDS: Maternal mortality/prevention \& control; Prenatal care; Women's health

1 Professora Livre-docente de Obstetrícia - Adjunta do Departamento de Ginecologia e Obstetrícia da Faculdade de Medicina de Botucatu da Universidade Estadual Paulista "Julio Mesquita Filho" - UNESP - Botucatu (SP), Brasil.

2 Professor Livre-docente de Obstetrícia - Associado do Departamento de Tocoginecologia da Faculdade de Ciências Médicas da Universidade Estadual de Campinas - UNICAMP - Campinas (SP), Brasil.

3 Presidente do Comitê de Mortalidade Materna da cidade de São Paulo (SP), Brasil.

Correspondência: Iracema de Mattos Paranhos Calderon

Rua Atílio Losi, 226 - Jd. Paraíso - 18 610-260 - Botucatu - SP - Telefone: (14) 3811-6227 - Fax: (14) 3882-1933 - e-mail: calderon@fmb.unesp.br 


\section{Introdução}

A razão de mortalidade materna (RMM) é indicador da qualidade de saúde, influenciada diretamente pelo grau de desenvolvimento econômicocultural-tecnológico de um país ou sociedade ${ }^{1}$. A Figura 1 apresenta as estimativas de RMM da Organização Mundial de Saúde (OMS) para o ano 2000, identificando risco elevado de morte materna (MM) nos países pobres, com dificuldades sociais importantes, em oposição aos países desenvolvidos, onde este índice não ultrapassa 50,0 mortes maternas/ 100.000 nascidos vivos $(\mathrm{NV})^{2}$.

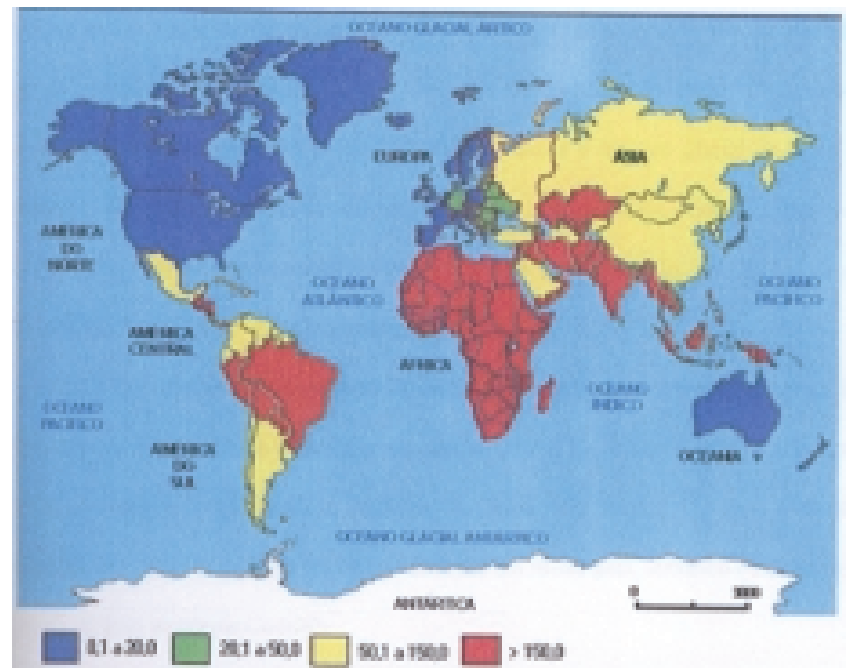

Figura 1 - Razão de mortalidade materna mundial, de acordo com a classificação de risco para mortalidade materna em baixo $(0,1-20,0)$, médio $(20,1-50,0)$, alto $(50,1-150,0)$ e muito alto $(>150,0)$.

Fonte: Maternal Mortality in 1995: WHO/UNICEF/UNFPA estimates - 2000. Elaborado por Vega (2004)

Cabe ressaltar que a RMM foi superestimada para o Brasil e não atinge os valores de 190/100.000 NV. Na última publicação do Ministério da Saúde do Brasil $^{3}$, a RMM oficial do país é de 55,8/1000.000 $\mathrm{NV}$, distribuída em 53,4 para a região Sudeste, 61,9 para a região Sul e 58,3 para região Centro-Oeste.

O sistema de coleta de dados sobre morte materna do Brasil é classificado no grupo E, ou seja, má qualidade dos dados e pela falta de pesquisa minuciosa e abrangente em todo o território nacional ${ }^{1}$. Com raras exceções, os índices são calculados a partir das MM declaradas, obtidas das declarações de óbito, não contemplando os casos mascarados ou não declarados. Assim, o Brasil ainda padece de dois sérios problemas: é país em desenvolvimento, com o ônus de todas as dificuldades inerentes a esta condição, e não tem sistema de registro adequado para os casos de MM.

Independentemente da dificuldade na avaliação da RMM, os dados oficiais sinalizam uma situação real - a falta de qualidade dos serviços de assistência à gestação, parto e puerpério. Isto não é exclusivo do Brasil, mas atinge todos os países em desenvolvimento, onde estão as gestantes mais necessitadas e com maior dificuldade de acesso a assistência de qualidade. As cinco principais causas de MM direta nestes países são as hemorragias (25\%), seguidas pelas infecções (15\%), as complicações do aborto (13\%), a eclâmpsia (12\%) e as distócias/obstruções no trabalho de parto $(8 \%)^{4}$.

Tal constatação tem motivado as principais organizações de saúde, apoiadas por instituições de pesquisa, a elaborarem guias assistenciais específicos, orientados pelos novos conceitos da medicina baseada em evidência, com comprovada efetividade na prevenção da morbimortalidade materna.

\section{As evidências cientificas no pré-natal}

A assistência pré-natal não pode prevenir as principais complicações do parto na grande maioria das mulheres destinadas a esta experiência - hemorragias, septicemias, obstruções do trabalho de parto. Mas certas intervenções durante a gravidez poderão, certamente, alterar e favorecer o prognóstico materno ${ }^{5}$. What Works: A Policy and Program Guide to the Evidence on Family Planning, Safe Motherhood, and STI/HIV/AIDS Interventions - Module 1 - Safe Motherhood ${ }^{6}$ e The Safe Motherhood Strategies Project ${ }^{7}$ são publicações recentes, embasadas em evidências científicas, que destacam práticas benéficas na assistência pré-natal para prevenir a morte materna. As estratégias mais importantes constituem um tripé com intervenções específicas relacionadas à promoção da saúde materna, à prevenção dos riscos e à garantia de suporte nutricional durante a gestação.

Promover a saúde materna contempla a recomendação do número ideal e da qualidade das consultas de pré-natal, o estabelecimento de programa de imunização materna e a prevenção, diagnóstico e tratamento das doenças intercorrentes da gestação. O manual técnico preparado pelo Grupo de Investigações de Estudo de Controle Pré-natal da $\mathrm{OMS}^{8}$ considera ideal a realização de quatro consultas no pré-natal e uma no período pós-parto. Na primeira visita, recomendada até a $16^{\underline{a}}$ semana de gestação, deverá ser avaliado o risco obstétrico por aplicação de formulário específico (Quadro 1). A detecção de qualquer risco implicaria atenção especializada, com exame/avaliação e seguimentos adicionais e, se necessário, a referência da atenção básica para um serviço de nível mais complexo. Importante destacar que o risco e a necessidade de referência para centros mais especializados deverão ser constantemente avaliados. $\mathrm{Na}$ ausência de risco, o acompanhamento pré-natal deverá seguir as recomendações para a atenção básica na assistência pré-natal (Quadro 2). 
Quadro 1 - Critériós para investigação do risco gestacional e classificação da gestante no componente básico do modelo de assistência pré-natal ${ }^{8}$.

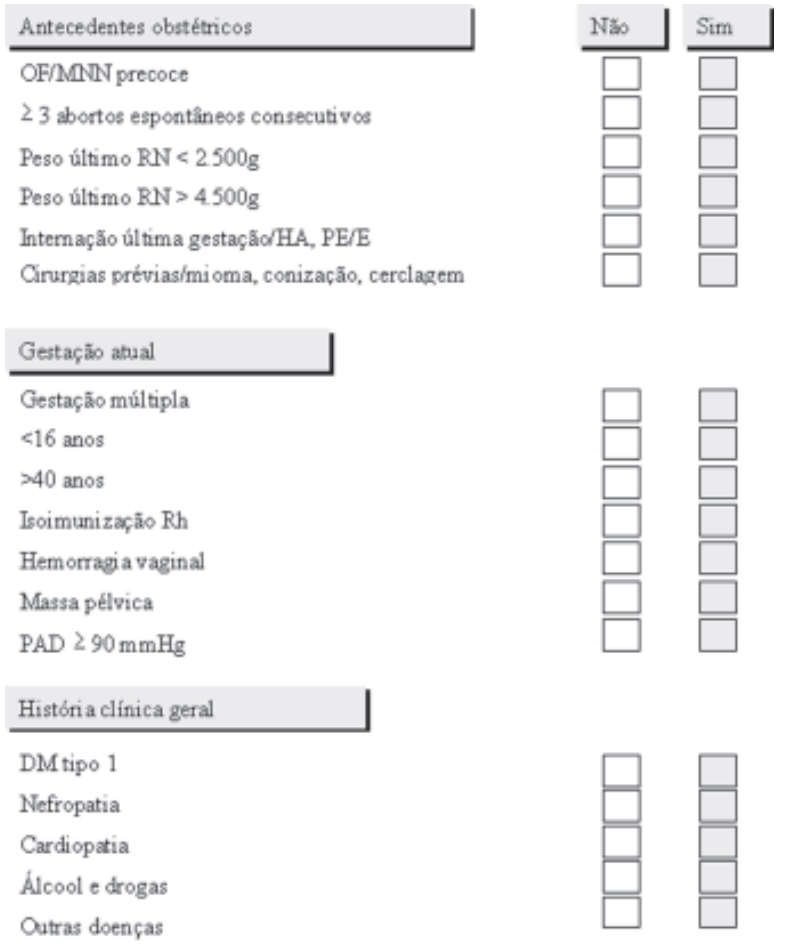

Especifique

Observação: Uma anotação SIM em qualquer uma das perguntas significa que a gestante não e elegivel para a assistencia pre-natal basica e devera ser individualizada e/ou referenciada para serviços especializados. Fonte: Manual para a prática de um novo modelo de assistência pré-natal ${ }^{8}$. Adaptado pelos autores. $O F=$ óbito fetal; $M N N=$ morte neonatal; $\mathrm{RN}=$ recém-nascido; $\mathrm{HA}=$ hipertensão arterial; $\mathrm{PE} / \mathrm{E}=$ pré-eclâmpsia/ eclâmpsia; $\mathrm{PAD}$ = pressão arterial diastólica; $\mathrm{DM}=$ diabete melito.

Quadro 2 - Componente básico do novo modelo de assistência pré-natal da OMS adaptado pelos autores.

\begin{tabular}{|c|c|c|c|c|}
\hline Procedimentos & \multicolumn{4}{|c|}{$\begin{array}{c}\text { Idade gestacional } \\
\text { (semanas) }\end{array}$} \\
\hline Investigar risco obstétrico & $X$ & $X$ & $X$ & $X$ \\
\hline Exame clínico & $X$ & $X$ & $X$ & $X$ \\
\hline Anemia grave clínica/níveis de $\mathrm{Hb}^{*}$ & $X$ & & $X$ & \\
\hline Exame obstétrico - IG; AU; BCF & $X$ & $X$ & $X$ & $X$ \\
\hline Exame ginecológico & $X$ & & & \\
\hline Pressão arterial & $X$ & $X$ & $X$ & $X$ \\
\hline Relação peso/altura & $X$ & & & \\
\hline Sorológicos sífilis/DST ${ }^{\star \star}$ & $X$ & & & \\
\hline Exame de urina (múltiplas tiras reagentes) & $X$ & & & \\
\hline Tipagem sangüínea - ABO; Rh & $X$ & & & \\
\hline VAT & $X$ & & $X$ & \\
\hline Suplementação ferro e ácido fólico & $X$ & $X$ & $X$ & $X$ \\
\hline Orientações emergência & $X$ & $X$ & $X$ & $X$ \\
\hline Ficha pré-natal/preencher & $X$ & $X$ & $X$ & $X$ \\
\hline Peso materno para as de baixo peso & & $X$ & $X$ & $X$ \\
\hline Proteinúria para nulíparas/PE prévia & & $X$ & $X$ & $X$ \\
\hline Orientações/planejamento do parto & & & $X$ & $X$ \\
\hline Orientações lactação/contracepção & & & $X$ & $X$ \\
\hline Apresentação pélvica/referência VCE & & & & $X$ \\
\hline
\end{tabular}

Avaliacão laboratorial dos níveis de $\mathrm{Hb}$ na primeira e terceira visitas de pré-natal, conduta também adotada pelo Manual Técnico do MS-Brasil/20009 "Solicitacão de sorologia tambem adotada pelo Manual Técnico do MS-Brasi/200i. "Solicitaçao de sorologia para sifilis/DST apenas na primeira visita de pré-natal, diferente da recomendação do Manual Tecnico do MS-Brasil/2000, que orienta repetir no terceiro trimestre sempre que os resultados forem negativos na primeira avaliação. IG = idade gestacional; AU = $\mathrm{VAT}$ = vacina antitetânica; $\mathrm{PE}$ = pré eclâmpsia; $\mathrm{VCE}$ = versão cefálica externa.
Para assegurar a qualidade da assistência pré-natal, a OMS recomenda: investigar o risco obstétrico; realizar exame clínico e obstétrico, com especial atenção à presença de anemia e avaliação da idade gestacional, altura uterina e batimentos cárdio-fetais; aferir os níveis pressóricos; reforçar e estimular a suplementação de ferro e ácido fólico; instruir a gestante sobre os sinais e os locais de atendimento de emergência e preencher a ficha de pré-natal de maneira adequada em todas as consultas de pré-natal. Além destes procedimentos, acrescenta na primeira consulta o exame ginecológico completo, o cálculo da relação peso/altura, a solicitação de exames laboratoriais básicos, como dosagem de hemoglobina (Hb), sorológico para sífilis/DST, urinálise e tipagem sangüinea ( $\mathrm{ABO}$ e $\mathrm{Rh}$ ) e a primeira dose da vacina antitetânica ${ }^{8}$.

O protocolo inclui também a avaliação de peso materno nas gestantes de baixo peso e de proteinúria nas nulíparas ou portadoras de préeclâmpsia prévia em todas as visitas subseqüentes à primeira consulta de pré-natal. A dosagem de $\mathrm{Hb}$ e a vacina antitetânica deverão ser repetidas na terceira consulta; orientações e planejamento do parto e recomendações sobre lactação e anticoncepção deverão ser iniciadas na terceira e reforçadas na última visita. Normatiza o diagnóstico de apresentação pélvica e encaminhamento para versão cefálica externa no termo da gestação (última consulta) e reforça o preenchimento e a instrução à gestante para que apresente a ficha de pré-natal completa no momento do parto ${ }^{8}$.

Por fim, destaca alguns pontos importantes: a) esta ficha não deverá substituir a história clínica, mas servir de controle para que estas metas básicas sejam cumpridas; b) a equipe técnica e os gestores de saúde não deverão medir esforços para a implementação de todas as atividades recomendadas; c) alguns documentos específicos já estão em andamento e deverão facilitar a implementação do novo modelo de assistência pré-natal ${ }^{9}$.

As evidências confirmam que a assistência pré-natal básica pode ser desenvolvida não só por médico-obstetra, mas por outros profissionais, como enfermeiros e enfermeiros-obstetras ${ }^{6}$. Um ensaio multicêntrico, envolvendo Argentina, Cuba, Arábia Saudita e Tailândia, confirmou resultados semelhantes na assistência pré-natal praticada por médicos obstetras e por pessoal treinado (enfermeiras-obstetras, enfermeiras e outros ${ }^{10}$.

No contexto da promoção da saúde materna, são incluídos programas de imunização materna contra as principais doenças, individualizadas por regiões ou países, e de prevenção, diagnóstico e tratamento das doenças intercorrentes mais co- 
muns da gestação ${ }^{6}$. Prevenir, diagnosticar e tratar a anemia materna, a hipertensão gestacional, a pré-eclâmpsia grave e eclâmpsia e os vários tipos de infecções intercorrentes na gestação e parto, incluindo as doenças sexualmente transmissíveis, as do trato urinário e o tétano do recémnascido, são intervenções efetivas de comprovada evidência científica ${ }^{7}$.

A suplementação de ferro $\left(\mathrm{Fe}^{++}\right)$e ácido fólico e a dosagem sérica de hematócrito e $\mathrm{Hb}$ têm comprovada evidência na prevenção da anemia materna (Figuras 2 e 3).

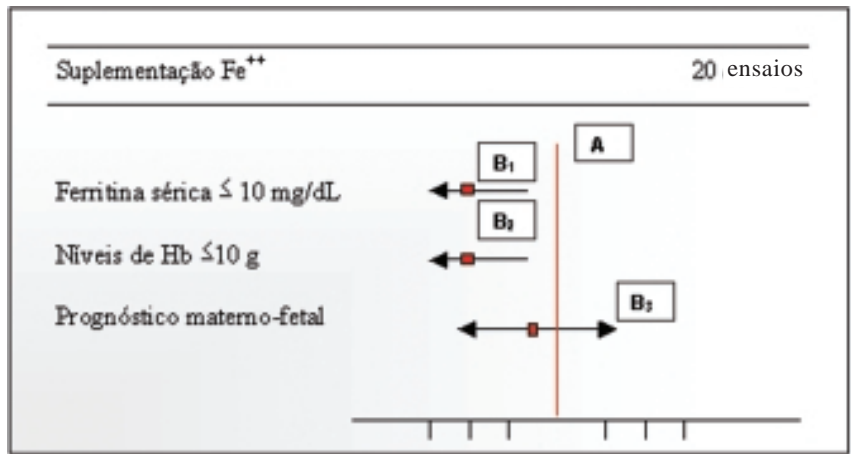

Figura 2 - Representação gráfica da revisão sistemática sobre suplementação de Fe na gestação ${ }^{11}$, adaptado pelos autores.

Representação gráfica de um resultado de metanálise: o eixo vertical $\mathrm{A}$ (linha vermelha) representa a linha da nulidade (ou do 1); as linhas/setas horizontais $\left(B_{1}, B_{2}\right.$ e $\left.B_{3}\right)$ representam o intervalo de confianca a $95 \%$ (IC 95\%) e o quadrado vermelho sobre as linhas/setas horizontais, o odds ratio de cada resultado avaliado.

Os resultados com IC 95\% limitados à esquerda da linha do 1 são interpretados como efeitos benéficos da intervenção; aqueles à direita da linha do 1 são considerados efeitos danosos, e os que incluem/ultrapassam esta linha indicam falta de qualquer tipo de efeito da intervenção avaliada.

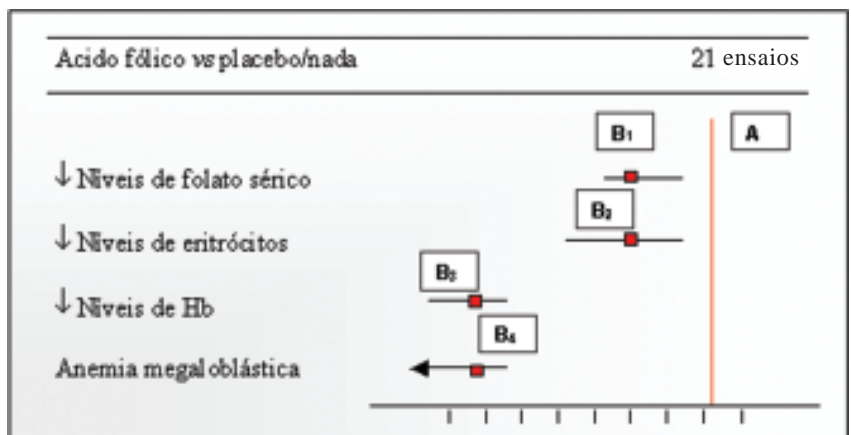

Figura 3 - Representação gráfica da revisão sistemática sobre suplementação de ácido fólico na gestação ${ }^{11}$, adaptada pelos autores.

Representação gráfica de um resultado de metanálise: o eixo vertical $A$ (linha vermelha) representa a linha da nulidade (ou do 1); as linhas/setas horizontais $\left(B_{1}, B_{2}, B_{3}\right.$ e $\left.B_{4}\right)$ representam o intervalo de confiança a $95 \%$ (IC95\%) e o quadrado vermelho sobre as linhas/setas horizontais, o odds ratio de cada resultado avaliado.

Os resultados com IC 95\% limitados à esquerda da linha do 1 são interpretados como efeitos benéficos da intervenção; aqueles à direita da linha do 1 são considerados efeitos danosos, e os que incluem/ultrapassam esta linha indicam falta de qualquer tipo de efeito da intervenção avaliada.

De acordo com a revisão sistemática da $\mathrm{Bi}$ blioteca Cochrane, a suplementação de ferro previne níveis baixos de $\mathrm{Hb}$ no parto e até seis semanas pós-parto, mas ainda faltam informações so- bre o prognóstico materno-fetal e dados específicos de populações com reconhecida deficiência de ferro; a suplementação de acido fólico favorece aumento dos niveis de $\mathrm{Hb}$ e folato no parto e até seis semanas após, mas não há evidências para avaliar qualquer outro efeito no prognóstico materno e fetal ${ }^{11}$.

A anemia associada à malária prejudica a evolução da gravidez e a malária per se pode favorecer ou agravar a anemia preexistente ${ }^{12}$. Para as regiões endêmicas, a profilaxia, o diagnóstico e o tratamento da malária são medidas benéficas e devem ser implementadas. Esquemas com sulfadoxina-pirimetamina são efetivos para a profilaxia durante a gestação, incluindo as gestantes HIV+, mesmo as de admissão tardia no pré-natal ${ }^{13}$. Para o tratamento da malária, a artemisina é superior à quinina na prevenção da morte materna nos quadros graves ou complicados, especialmente na resistência a múltiplas drogas, mas favorece a recorrência dos episódios de malária. A associação de artemisina com outros agentes, como a mefloquina ou a lumofantrina, é efetiva para o tratamento e previne a recorrência, apesar do inconveniente do custo elevado ${ }^{6}$.

As evidências comprovam que a imunização da gestante contra o tétano é a mais simples e benéfica medida para reduzir a mortalidade neonatal e a ocorrência de tétano materno, responsável por pelo menos 5\% das MM nos países em desenvolvimento. Outras recomendações de comprovada efetividade incluem a prevenção, o diagnóstico e o tratamento de infecções parasitárias intestinais e de tuberculose. O tratamento da parasitose intestinal melhora a saúde materna, previne a anemia e aumenta o peso dos recémnascidos; o controle da tuberculose reduz o número de $\mathrm{MM}$, principalmente naquelas portadoras de $\mathrm{HIV}^{6}$.

Quanto à pré-eclâmpsia, não está claramente definido o tratamento ou o tipo de intervenção efetiva para prevenir sua evolução para eclâmpsia. Para alguns, o melhor seria a indução do parto, mas isto pode não ser possivel em gestações precoces. Nestes casos, o repouso e o monitoramento constante seriam opções aceitáveis ${ }^{6,14,15}$.

Na publicação do The Safe Motherhood Strategies Project, a referência para um serviço de atenção terciária nos quadros de pré-eclâmpsia grave e a administração de sulfato de magnésio para a eclâmpsia foram as únicas medidas com evidência científica demonstrada na redução da MM. Os autores destacaram, ainda, a falta de evidências sobre a ação de agentes antiplaquetários na prevenção da pré-eclâmpsia e sobre o uso de anti-hipertensivos no tratamento da pré- 
eclâmpsia leve. Também não há evidências favoráveis à suplementação de zinco, magnésio, vitamina A e cálcio na pré-eclâmpsia ${ }^{7}$.

Mais recentemente, a revisão sistemática da Biblioteca Cochrane demonstrou que a suplementação de cálcio previne a hipertensão arterial e a pré-eclâmpsia, com melhores resultados nas populações de risco ou com deficiência nutricional deste mineral. Entretanto, ainda não há evidência a respeito da dose mais adequada e de outros desfechos maternos e fetais ${ }^{16}$. Esta mesma fonte confirmou que o sulfato de magnésio, comparado ao placebo, à ausência de tratamento ou à fenitoína, é melhor na prevenção da eclâmpsia e, provavelmente, na redução do risco de morte materna. Entretanto, os revisores advertem para a ocorrência de $25 \%$ de efeitos colaterais, principalmente tremores musculares, e para a necessidade de mais investigações sobre o prognóstico neonatal ${ }^{17}$.

O Program for Appropriate Technology in Health define como prioridade para a prevenção de morte materna relacionada à eclâmpsia/pré-eclâmpsia: padronização de guias e treinamento da equipe técnica para a implementação universal do sulfato de magnésio; realização de ensaios clínicos controlados para avaliar os efeitos da suplementação de vitaminas A e E e de cálcio nas populações deficientes, além da avaliação do custo-benefício de fitas reagentes para investigação de proteinúria. Entre outras, prioriza o desenvolvimento de protocolos efetivos incluindo o uso de drogas para o preparo do colo (misoprostol), o treinamento da equipe profissional e a integração dos cuidados pósaborto no protocolo básico de assistência. Por fim, destaca a importância de normatização dos sistemas de referência para os casos de emergência obstétrica $^{18}$.

\section{Conclusões}

A nossa realidade está muito aquém das atuais propostas tecnológicas para a prevenção da MM. No Brasil, este sério problema de saúde pública está atrelado às subnotificações, às condições de alto risco para $\mathrm{MM}$, a maioria delas de causas diretas e evitáveis, e à falta de implementação de estratégias efetivas. Fatores culturais, médicoprofissionais e político-sociais são predominantes e facilmente reconhecidos, mostrando as iniqüidades existentes ${ }^{19-22}$. A estruturação do planejamento familiar, incluindo prevenção da gravidez de alto risco e daquelas não desejadas, enfrentamento crítico do problema do aborto e re- dução global das taxas de cesárea ainda são prioridades na assistência à saúde da mulher brasileira $^{23}$. A operacionalização efetiva destas prioridades deverá melhorar o panorama da MM em nosso país.

\section{Referências}

1. Vega CEP. Mortalidade materna na cidade de São Paulo de 1995 a 1999, com ênfase em hipertensão arterial. Rev Bras Ginecol Obstet [serial on the Internet]. 2004 [citado 21 Maio 2006];26(8):672. Disponível em http://portal.prefeitura.sp.gov.br/ secretarias/saude/mulher/0007.

2. WHO/UNICEF/UNFPA. Maternal mortality in 2000: estimates developed by WHO, UNICEF, UNFPA. WHO/WQ16 [homepage da Internet]. Geneva: World Health Organization; 2004 [citado 2005 Ago 2]. Disponivel em: http://www.who.int.

3. Ministério da Saúde. Fundação Nacional de Saúde. Centro Nacional de Epidemiologia. Fundação IBGE. Anuário 2001 [homepage da Internet]. Brasília; 2001 [citado 2005 Ago 2]. Disponível em: http:// www.datasus.gov.br.

4. WHO. Reduction of maternal mortality: a joint WHO/UNFPA/UNICEF [homepage da Internet]. Geneva: The World Bank Group; 2004 [citado 2005 Ago 2]. Disponivel em: http://www.who.int.

5. Gelband H, Liljestrand J, Nemer N, Islam M, Zupan $\mathrm{J}$, Jhan P. The evidence base for interventions to reduce maternal and neonatal mortality in low and middle-income countries. Geneva: WHO; 2001. (Commission on macroeconomics and health working paper $\mathrm{n}^{\mathrm{0}} 5$ ).

6. Gay J, Hardee K, Judice N, Agarwal K, Fleming K, Hairston A, et al. What works: a policy and program guide to the evidence on family planning, safe motherhood, and STI/HIV/AIDS interventions. Module 1 - Safe motherhood. Policy project [homepage on the Internet]. Washington; 2003 [cited 2005 Jun 22]. Available from: http:// www.policyproject.com/pubs/generalreport/ SM_WhatWorksps2.pdf.

7. De Brouwere V, Van Lerberghe W. Safe motherhood strategies: a review of the evidence [homepage on the Internet]. Antwerp: ITP Press; 2001 [cited 2005 Jun 22]. (Studies in health services organizations \& policy, 17). Available from: http://www.itg.be/itg/ GeneralSite/generalpage.asp?wpid.

8. Villar J, Bergsjø P. Ensayo clínico aleatorizado de control prenatal de la OMS: Manual para la puesta en práctica del nuevo modelo de control prenatal [homepage da Internet]. Genebra: Grupo de Investigación del Estudio de Control Prenatal de la OMS; 2003 [citado 2006 Maio 12]. Available from: http://who.int/reproductive-health/rhl. 
9. Ministério da Saúde. Secretaria de Políticas Públicas de Saúde. Assistência pré-natal. $3^{a}$ ed. Brasília: SPS/Ministério da Saúde; 2000.

10. Villar J, Carroli G, Khan-Neelofur D, Piaggio G, Gülmezoglu M. Patterns of routine antenatal care for low-risk pregnancy. Cochrane Database Syst Rev. 2005;(2):CD000934.

11. Mahomed K. Iron supplementation in pregnancy. Cochrane Database Syst Rev. 2005;(2):CD000117.

12. AbouZahr C. Maternal mortality overview. In: Murray CJL, Lopez AD, editors. Health dimensions of sex and reproduction. Global burden of disease and injury series. Geneva: World Health Organization; 1998. v. 3, p.111-64.

13. Shulman CE, Dorman EK, Cutts F, Kawuondo K, Bulmer JN, Peshu $\mathrm{N}$, et al. Intermittent sulphadoxine-pyrimethamine to prevent severe anaemia secondary to malaria in pregnancy: a randomised placebo-controlled trial. Lancet. 1999;353(9153):632-6.

14. Rosenberg K, Twaddle S. Screening and surveillance of pregnancy and hypertension - an economic approach to the use of daycare. Baillieres Clin Obstet Gynaecol. 1990;4(1):90-107.

15. McDonagh M. Is antenatal care effective in reducing maternal morbidity and mortality? Health Policy Plann. 1996;11(1):1-15.
16. Atallah AN, Hofmeyr GJ, Duley L. Calcium supplementation during pregnancy for preventing hypertensive disorders and related problems. Cochrane Database Syst Rev. 2005;(2):CD001059.

17. Duley L, Gülmezoglu AM, Henderson-Smart DJ. Magnesium sulphate and other anticonvulsants for women with pre-eclampsia. Cochrane Database Syst Rev. 2005;(2):CD000025.

18. Tsu VD. New and underused technologies to reduce maternal mortality. Lancet. 2004;363(9402):75-6.

19. Cecatti JG, Pires HMB, Goldenberg P. Cesárea no Brasil: um direito de opção? In: Galvão L, Díaz J, editores. Saúde sexual e reprodutiva no Brasil: dilemas e desafios. São Paulo: Hucitec/Population Council; 1999. p. 237-54.

20. Tanaka ACA. Mortalidade materna: reflexo da má qualidade e da desintegração dos serviços de saúde. J Rede Saúde. 2000;20:5-8.

21.Laurenti R, Jorge MHPM, Gotlieb SLD. Reflexões sobre a mensuração da mortalidade materna. Cad Saúde Pública. 2000;16(1):23-30.

22.Laurenti R, Mello-Jorge MHP, Gotlieb SLD. A mortalidade materna nas capitais brasileiras: algumas características e estimativa de um fator de ajuste. Rev Bras Epidemiol. 2004;7(4):449-60.

23. Faundes A, Hardy E. Illegal abortion: consequences for women's health and the health care system. Int J Gynaecol Obstet. 1997;58(1):77-83. 